\title{
«Comme si nous étions à la contredanse » : chorégraphies utopiques chez Claire de Duras et George Sand
}

La danse s'intègre dans les sociétés utopiques en tant que signe d'euphorie et outil éducatif. La danse saisit les sens et produit un plaisir naturel que les Utopiens de Thomas More classeraient comme plaisir physique (2003, p. 77). Selon Charles Fourier, « un peuple très poli, bien chantant et dansant » avance beaucoup, car ce raffinement permet aux individus d'acquérir "l'aplomb, la mesure et l'unité » nécessaires pour accomplir des tâches manuelles et gérer leurs passions $(1829$, p. 124, p. 260). Considérant la danse comme exercice essentiel à l'éducation des enfants, Fourier encourage la phalange, son concept d'une société harmonieuse, à exécuter des «manœuvres chorégraphiques " pour qu'elle soit «manœuvrière comme des danseurs et comparses d'opéra " (p. 136). En plus de former les individus et de produire du plaisir, la danse peut esquisser des rapports humains et des manières d’être qui contrecarrent les idéologies dominantes, car les danseurs vivent des expériences spatiotemporelles divergeant de celles de la vie quotidienne. La danse exprime ainsi des mouvements vers des idéaux. En 1936, Paul Valéry définit la danse comme " action transposée dans un monde, dans une sorte d'espace-temps, qui n'est plus tout à fait celui de la vie pratique » (2011, p. 83). Cet espace-temps peut être à la fois un ou-topos qui n'existe nulle part, ou qui existe dans un « monde neutralisé, le même monde et cependant Autre Monde ", et un eu-topos où la joie règne (Marin, 1973, p. 72). Marquée d'une certaine ambiguïté, la scène de danse devient utopie en présentant " une figure, un schème de l'imagination, une fiction "produite-productrice" "

Tessa Ashlin Nunn - membre associée de l'IHRIM. Adresse de correspondance : IHRIM, 15 parvis René Descartes, 69342 Lyon Cedex 7, France ; e-mail : tessanunn@gmail.com

ORCID iD : https://orcid.org/0000-0003-1984-8645 
(p. 41). Dans la mesure où elle porte sur ce qui peut être vu ou vécu, la danse propose des relations humaines possibles, voire des sociétés à construire.

Dans la littérature, l'atemporalité utopique de la danse donne lieu aux rapports amoureux interdits dans le temps du récit. Des chercheurs constatent, après le foisonnement d'œuvres utopiques au XVIII ${ }^{\mathrm{e}}$ siècle, soit une pénurie d'utopie littéraire, soit un nouveau genre d'utopie basé sur les sciences sociales pendant la première moitié du XIX ${ }^{e}$ siècle. Pourtant, à cette époque, des danseurs mettent en scène des utopies chorégraphiques aux bals et dans des textes. Modèles cinétiques, rythmiques et visuels de la cohésion sociale, les contredanses, ou country dances, représentent, depuis leur apparition en Angleterre au XVI ${ }^{\mathrm{e}}$ siècle, une communauté sans distinctions, sans exigences. Ces danses jouent un rôle important dans des œuvres de Sophie Cottin, Germaine de Staël, Honoré de Balzac et d'autres. Nous limitons la présente étude aux scènes de contredanses dans deux romans : Édouard (1825) de Claire de Duras et Le Compagnon du Tour de France (1840) de George Sand. Dans ces récits, le mélange social semble possible au moment des contredanses, moment où les protagonistes peuvent s'unir avec ceux qu'ils aiment véritablement. Toutefois, cette liberté n'existe que dans l'espace de la danse.

En raison de ses figures ainsi que sa place dans l'évolution chorégraphique en Europe, la contredanse est la danse utopique par excellence. Chez Duras et Sand, les contredanses crayonnent une société désirée, mais pas encore réalisable à cause des préjugés enracinés dans les gestes quotidiens de la société actuelle. Après un survol de l'histoire de ces danses, nous nous interrogeons sur leurs liens avec l'anglophilie et l'esprit révolutionnaire dans Édouard. Ensuite, nous montrons comment elles deviennent une métaphore pour une société sans classe chez Sand. Existant dans le monde réel des romancières, les contredanses créent des moments utopiques, où les règles sociales basculent, dans des romans que nous ne qualifions pas d'utopiques.

\section{Danser sur un pied d'égalité}

$\mathrm{Au} \mathrm{XVIII}{ }^{\mathrm{e}}$ siècle, les contredanses à la cour française entament un changement de la politique corporelle en substituant des figures dans lesquelles tous dansent sur un pied d'égalité à celles qui ne mettent en lumière que certains danseurs. Ces danses contribuent donc à remplacer « le corps du roi » par celui de la société en tant que symbole de la nation (Foucault, 1975, p. 2). L'esprit des contredanses s'aligne sur celui du siècle des Lumières « qui à la rigoureuse ordonnance du jardin à la française, symétrique et statique, se met à préférer le foisonnement faussement désordonné du parc à l'anglaise » (Granger, 2019, p. 16). Grâce à la musique simple mais entraînante des contredanses, leurs pas et leurs figures semblent s'exécuter librement dans un état joyeux.

Certains maîtres à danser se plaignent du fait que les contredanses font négliger la belle danse née sous le règne de Louis XIII, car elles sont « trop badines et folâtres 
pour des personnes du bel air» (Pauli, 1756, p. 66) et « ne sont point dans la bienséance " (Rameau, 1748, p. 108). Contrairement à la danse baroque, la contredanse est «mouvement, non position, et les figures qu'elle propose aux danseurs sont autant de parcours à éprouver, plutôt que des dessins à contempler " (Guilcher, 1998, p. 84). Pendant la première moitié du XVIII e siècle, la belle danse et les contredanses coexistent aux bals, puis la danse baroque cède sa place aux contredanses qui, à la fin du siècle, atteignent l'acmé de leur popularité. Bien que la valse finisse par les devancer, elles se voient régulièrement exécutées aux fêtes pendant la première moitié du XIX ${ }^{e}$ siècle.

La contredanse est révolutionnaire dès lors qu'elle reconstruit le visible et le pensable. Dans ses Mémoires, le duc de Saint-Simon décrit un bal à la cour d'Espagne durant lequel la reine espagnole danse avec tout le monde : " comme aux contredanses on se mêle, et, suivant l'ordre de la contredanse, chacune se trouve danser avec tout ce qui danse, l'un après l'autre, et se retrouve au bout avec son meneur, la reine y dansait de même avec tout le monde " $(1830$, p. 86$)$. Au cours de la danse, toutes les divisions sociales se dissolvent.

Les figures tracent " une image-idée d'une société alternative » dans laquelle chaque individu s'épanouit sans piétiner sur la liberté d'autrui (Baczko, 1989, p. 15). Le philosophe allemand Friedrich Schiller a recours aux contredanses anglaises afin de définir ce qu'il faut faire au sein des relations sociales : " ménagez la liberté d'autrui » («Schone fremde Freiheit») et «montrez votre liberté» («Zeige selbst Freiheit») (1971, p. 54). D’après Schiller, les danseurs laissent leurs places aux suivants, comme s'ils pensaient toujours aux autres sans s'oublier eux-mêmes. En effet, chaque participant occupe toutes les places comme si tout l'espace était un bien commun, de sorte qu'une coexistence sans possession s'établisse.

\section{2. Édouard : danser en Angleterre}

Duras écrit Édouard, pendant la Restauration, dans l'objectif de «montrer l'infériorité sociale telle qu'elle existait avant la Révolution» (cité dans Pailhès, 1910, p. 462). À l'instar du héros goethéen, Édouard s'abandonne à la rêverie. Fils d'un bourgeois lyonnais, le héros éponyme déménage à Paris afin de devenir avocat, sous la protection du maréchal d'Olonne, et tombe amoureux de Natalie de Nevers, la fille de ce dernier. Après la mort du père d'Édouard, le maréchal s'engage à veiller sur lui comme s'il était son fils. Au moment où le maréchal découvre qu’Édouard aime sa fille, il le bannit de sa maison.

Comme dans Corinne de Germaine de Staël et Valérie de Barbara Julianne de Krüdener, le héros se cache pour regarder danser sa bien-aimée, ce qui transforme les danses aux bals en spectacles. Puisque Édouard ne peut assister au bal de l'ambassadeur d'Angleterre en tant qu'invité, à cause de son rang social, il s'y trouve comme spectateur dissimulé dans les gradins. Là, il observe furtivement la fête : « Je n’avais 
jamais vu danser madame de Nevers, et j'avais un violent désir de la voir, sans en être $\mathrm{vu}$, à une de ces fêtes où je me la représentais si brillante. On pouvait aller à ces grands bals en tant que spectateur ; cela s'appelait aller en beyeux " (Duras, 2007, p. 138139). La locution « en bayeux » vient de l'appellation « les Beyeux de Saint-Quentin » qui fait allusion à la tendance des habitants de Saint-Quentin à scruter les étrangers, le verbe picard béer signifiant regarder ou "rêver les yeux ouverts" (Matinées sénonoises, 1789, p. 232). Les spectateurs en bayeux, "séparés du reste de la société " et entourés "des personnes d'un rang inférieur, et qui ne pouvaient aller à la cour ", doivent acheter des billets afin d'admirer le grand monde dansant (Duras, 2007, p. 138-139). Fête théâtralisée, le bal garantit ainsi la hiérarchie sociale, tandis qu'une " fête complètement déthéâtralisée » donnerait lieu à la possibilité d'une société sans distinction de classe (Ozouf, 1976, p. 342).

En raison de cet aménagement, les bornes sociales se traduisent en obstacles tangibles. Bien que Madame de Nevers n'aperçoive pas Édouard, le Duc de L. le voit et l'appelle afin qu'elle le remarque. S'approchant, Édouard demeure séparé d'elle par une clôture devant les gradins, « barrière qui isolait les spectateurs de la société : triste emblème de celle qui nous séparait pour toujours!» (Duras, 2007, p. 140). La configuration de l'espace différencie ainsi ceux qui dansent de ceux qui regardent, qui se soumettent au pouvoir des acteurs ayant accès à la scène politique.

Situé avant 1783, le récit représente l'Angleterre comme sanctuaire démocratique par rapport à la France de l'ancien régime. Étranger à cet ordre, l'ambassadeur anglais invite Édouard à participer au bal. Ce dernier franchit la barrière et s'assoit sur un banc près des gradins, mi-chemin entre les spectateurs et les danseurs. Là, il rêve de partir en Angleterre où il croit que son talent et son courage compenseraient ses origines modestes. Critique de la hiérarchie rigide qui domine en France, Édouard se plie pour autant aux lois sociales.

Grâce aux contredanses, le songe de vivre dans une société plus ou moins démocratique se réalise momentanément. Révélateur de la peur d'Édouard de lutter pour ce rêve, le dialogue entre Madame de Nevers et lui, qui forme une invitation à danser, présage son refus de mariage lorsqu'elle propose de quitter son milieu aristocratique afin de s'unir à lui :

Mais ne dansez-vous pas? me demanda-t-elle. - Je crains que cela ne soit inconvenable, lui dis-je. -Pourquoi donc ? reprit-elle; puisque vous êtes invité, vous pouvez danser, et je ne vois pas ce qui vous en empêcherait. Et qui inviterez-vous? ajouta-t-elle en souriant. - Je n’ose vous prier, lui dis-je ; je crains qu'on ne trouve déplacé que vous dansiez avec moi. Encore s’écria-t-elle; voilà réellement de l'humilité fastueuse. - Ah ! lui dis-je tristement, je vous prierais en Angleterre. (p. 141)

Duras s'appuie sur un lien bien établi entre la contredanse et l'Angleterre mis en évidence par l'anglophilie foisonnante au tournant du XVIII ${ }^{\mathrm{e}}$ siècle. Les contredanses anglaises arrivent à la cour française vers la fin du XVII ${ }^{e}$ siècle et y évoluent selon 
les mœurs de la cour ${ }^{1}$. Dans ses notations de contredanses anglaises offertes à Louis XIV en 1685, André Lorin explique qu'il opte pour les termes Seigneur et Dame quoique les Anglais désignent les danseurs man et woman. En outre, Lorin invente un rôle pour le roi en le nommant celui qui décide à quel moment les contredanses auront lieu. Lors de la Révolution de 1789, elles deviennent toutefois symboles de l'esprit révolutionnaire et d'un certain engouement pour un modèle gouvernemental similaire au système anglais.

Depuis La Princesse de Clèves, la première danse dans le roman européen fait souvent allusion à la possibilité d'un premier rapport sexuel. Dans Édouard, comme dans le roman de Madame de Lafayette, les sentiments amoureux lors d'une danse n'amènent pas au mariage à cause des notions de bienséance, voire de devoir. Cependant, les effleurements de mains et la proximité corporelle donnent l'impression d'une véritable union amoureuse. Ce premier serrement de main, écrit Stendhal, produit le "plus grand bonheur que puisse donner l'amour» (1833, p. 160). Touchant la main de sa bien-aimée, Édouard réalise une aspiration : « je pris sa main, sa main que je n'avais jamais touchée ! et nous nous mîmes à une contredanse » (Duras, 2007, p. 142). La veille du bal, Madame de Nevers sort et oublie son gant qu'Édouard embrasse comme un objet fétichique qui, d'une perspective freudienne, rend le désir sexuel moralement permissible ainsi que facilement accessible. D'une façon similaire, la danse autorise des relations et des mouvements interdits dans la vie quotidienne mais licites durant le temps presque irréel de la danse.

Cette scène de bal bascule l'ordre social ainsi que les normes dictant le comportement féminin. Madame de Nevers franchit les règles de bienséance dans le but de se rapprocher d'Édouard dont la classe sociale le prive de supériorité masculine. Après qu'Édouard lui déclare être incapable de l'inviter, Madame de Nevers le prie de danser. Contraint par sa position inférieure d'accepter, il exécute une contredanse avec elle. Quoiqu'il se sente émasculé, il admire sa générosité, car il tient son invitation audacieuse pour un acte de charité : «Qu'elle était bonne et généreuse à ce bal ! elle a voulu danser avec moi, pour me relever à mes propres yeux, pour me consoler de tout ce qu'elle sentait bien qui me blessait » (p. 143). En même temps, cette hardiesse le mène à réfléchir sur le rôle de la femme : «Mais est-ce d'une femme ? est-ce de celle qu'on aime qu'on devrait recevoir protection et appui ? Dans ce monde factice tout est interverti, ou plutôt c'est ma passion pour elle qui change ainsi les rapports naturels » (p. 143). En répudiant les préceptes sociaux, Madame de Nevers lui permet de non seulement envisager mais de goûter un monde radicalement différent. Mariée à un vieillard à l'âge de 16 ans puis veuve à 20 ans, elle revendique le droit de choisir l'homme qu'elle épousera. Tandis qu'Édouard rêve de fuir une société inique, elle tente de la transformer.

1. À partir de 1705, des contredanses exclusivement françaises, telles que le cotillon en forme de carré, voient le jour. 
Les contredanses incarnent des rapports homme-femme légèrement plus égalitaires et le rêve de briser le joug des classes. Sans laisser une trace palpable, ces danses dessinent des illusions qui ne demeurent que dans la mémoire corporelle. Pourtant, elles suscitent des réflexions sur les rapports censés être naturels et la possibilité de les altérer.

\section{Le Compagnon du Tour de France: danser dehors et hors des normes}

L'idéalisme sandien, explique Naomi Schor (1993), se présente comme une quête à la perfectibilité proposant des changements véritablement possibles. Il s'agit du « réel de l'utopie » qui incite l'esprit révolutionnaire à vouloir «structurer autrement la société » (Riot-Sarcey, 1998, p. 265). Contrariée de seulement transmettre les doctrines sociales à la mode, Sand en fait une synthèse originale qui anticipe « sur l'évolution sociale, sur la formation de la classe moyenne [...] sur l'avènement futur d'une société de loisirs " (Sylvos, 2015, p. 1220). Tout au long de sa carrière, Sand envisage des utopies champêtres en idéalisant les paysans dépourvus de l'artifice qui entache le milieu citadin.

Le Compagnon du Tour de France, publié en 1840 sans succès dû aux critiques de son pédantisme, représente les sociétés artisanales en 1823 et, comme dans plusieurs romans de Sand, une histoire d'amour entre personnages de classes différentes. Les ouvriers Pierre et Amaury, appelé « le Corinthien », tombent amoureux respectivement de Yseult de Villepreux et de la marquise Joséphine des Frenays. Tous les dimanches au château de Villepreux, les ouvriers, les paysans et la famille aristocratique se rassemblent dehors pour danser. Ces assemblées évoquent celles de Clarens après les vendanges, dans Julie, ou la nouvelle Héloïse de Rousseau, pendant lesquelles les maîtres et les paysans dansent ensemble sous le règne d'une " douce égalité » qui « rétablit l'ordre de la nature » (Rousseau, 1845, p. 694). Ces bals font partie de la mission du maitre qui s'égale momentanément aux domestiques « en sorte qu'ils pensent vouloir tout ce qu'on les oblige de faire ", sans qu'ils soient tentés d'imiter leur maitre (p. 514).

La danse constitue un refuge en libérant ses participants des contraintes de créer quelque chose de durable. Lorsque les auteurs des utopies, notamment Thomas More et Tammaso Campanella, abordent la question du travail, ils traitent surtout du rapport au temps. Les heures consacrées à la danse constituent un "précieux far niente ", pour emprunter le terme de Rousseau (1948, p. 77). Il ne s'agit pas de la paresse mais de "la jouissance de l'otium ", le temps où la hiérarchie des occupations et le souci de produire s'abolissent (Rancière, 2011, p. 68). Cet état suspensif ouvre la voie à la rêverie. Sans lien avec le passé ni le futur, un moment illusoire s'établit.

Débarrassant les paysans et les artisans de l'impératif de travailler, les assemblées dansantes posent une menace au fonctionnement social, d'après certains membres 
du clergé. Dans le roman, le comte de Villepreux convainc le curé de lever l'interdiction sur la danse le dimanche, force de lui donner des « cadeaux pour sa cave et pour son église " (Sand, 1988, p. 218). Sand fait probablement allusion au tract de PaulLouis Courier " Pétition pour des villageois qu'on empêche de danser », écrit en 1822 dans le but d'autoriser les assemblées dominicales à Azay-sur-Cher. À la fin du roman, un nouveau curé s'installe et interdit derechef la danse qu'il assimile à l'adultère et au libéralisme. Décidément, la force politique de la danse ne passe pas inaperçue.

Ayant lieu à l'ombre d'un vieux chêne, les danses en plein air paraissent, au premier abord, inviolées par les efforts civilisateurs qui donnent naissance aux préjugés. En effet, « dans la neutralité salubre d'un espace non cloisonné, toutes les distinctions " semblent disparaître loin des sites marqués par les régimes de pouvoir (Ozouf, 1976, p. 209). Ces moments festifs présentent néanmoins les classes ouvrière et paysanne comme des groupes déterminés au lieu de les représenter comme des individus capables d'améliorer leur situation. La convenance des assemblées dépend de l'effacement de l'individualité des prolétaires. Le comte déclare que le " peuple est grand et beau comme masse, il est misérable et chétif comme individu » (Sand, 1988, p. 562). En revanche, les scènes de danse, décrites de la perspective des danseurs, font ressortir la communauté d'individus qui agissent ensemble. Grâce à ce partage de lieu et de temps, "un être-ensemble sans assemblage » se construit (Nancy, 2001, p. 43).

Même si les danseurs de classes différentes s'entremêlent, les distinctions sociales persistent dans les détails. Au début, la marquise Joséphine des Frenays prend toujours son cousin Raoul comme partenaire. Encore qu'elle danse avec chaque participant, peu importe son danseur initial, elle n'ose toucher la peau des paysans. Sa main gantée rappelle à tous que les gens des classes inférieures la répugnent :

[elle] n'avait pas dédaigné de mettre sa petite main dans celle du paysan qui lui faisait visà-vis à la chaîne anglaise. Mais cette main était couverte d'un gant, ce qui parut fort injurieux à la plupart des danseurs, et ce qui les empêcha de l'inviter. (Sand, 1988, p. 219-220)

La vanité de Joséphine cache sa position contestable. Née " une vraie paysanne » et mariée à un marquis, elle surjoue ses airs de supériorité quoiqu'elle se remémore nostalgiquement son enfance bucolique (p. 285). Vu que le contact peau à peau confirme vivement l'humanité commune, les gants lui évitent de reconnaître ses affinités avec le peuple.

Les paysans et les ouvriers ne sont guère les seuls à profiter des assemblées. Dans ce que Jean-Louis Cabanès appelle une " comédie du libéralisme », le comte les rend possibles et orchestre l'apparence d'une communauté sans classes tout en renforçant son pouvoir $(2019$, p. 31$)$. Il assiste aux danses à l'effet de «familiariser les bonnes gens avec sa présence seigneuriale »(Sand, 1988, p. 219). Après que le comte interdit à Raoul de participer aux danses, car son badinage amoureux avec les paysannes risque de brouiller le spectacle utopique, Joséphine regrette de ne plus dan- 
ser. Le comte commande donc à Amaury de danser un cotillon avec elle : «Allons, offre-lui la main, et en place pour la contredanse ! c'est moi qui vais crier les figures » (p. 220). À la fois une contredanse et un jeu, le cotillon se distingue par le rôle du cotillonneur qui annonce les figures à danser. Comme un bon souverain, le cotillonneur doit " posséder une autorité morale sur les autres danseurs pour se faire obéir, avoir la même sollicitude pour tous et ne marquer de préférence à personne " (Giraudet, 1900, p. 86).

L'artisan et la marquise font un couple improbable, pourtant la danse éveille leur attirance mutuelle. Bien que ce soit la première fois qu'il danse avec une marquise, Amaury se résout à la faire « danser tout aussi bien qu'un autre» (Sand, 1988, p. 220). En revanche, Pierre se lance dans un spectacle de virtuosité pour la déstabiliser :

le Corinthien, qui, malgré son courage intérieur, n’avait pas encore osé la regarder en face, s'aperçut que cette reine du bal était si troublée qu'elle s'embrouillait dans les figures. Il n'y comprit rien d'abord, et, voulant l'aider à reprendre sa place sans être atteinte par les ronds-de-jambe impétueux du Berrichon [Pierre], il osa, mais sans aucun autre sentiment que celui d'une déférence naturelle, placer sa main sous le coude de la marquise pour l'empêcher de tomber. Ce coude nu entre une manche courte et une mitaine de soie noire était si rond, si mignon et si doux, que le Corinthien ne le sentit pas d'abord, et que, voyant le Berrichon lancé dans une pirouette irréfrénable et la marquise chanceler, il lui serra le coude pour la remettre en équilibre. Mais cette pression fut électrique. (p. 220)

Cet effleurement de peau attise les passions et souligne les affinités humaines entre les deux danseurs. La danse et la musique engendrent ainsi un espace-temps onirique :

le violon n'eut pas plutôt donné le signal de la contredanse suivante qu'il se retrouva, comme par magie, auprès de madame des Frenays, et que la main de celle-ci était dans la sienne. De quelle formule s'était-il servi pour l'inviter de nouveau, et comment l'avait-il osé ? Il ne le sut jamais. Un nuage flottait autour de lui, et il agissait comme dans un rêve. (p. 220)

Ce couple se forme et sépanouit dans la danse. Chaque dimanche, ils se retrouvent pour recréer cette union passionnée : "leurs mains se touchaient, leurs haleines se confondaient, et leurs regards se cherchaient pour se fuir et pour se chercher encore » (p. 220-21). Joséphine, auparavant méprisante, tente de gagner l'approbation des ouvriers pour faire croire à Amaury que leur relation existe véritablement au sein d'une communauté fugace. Au moment où la danse renverse les normes, les danseurs se transforment sous l'influence de cette nouvelle manière d'être.

L'instant dansant constitue un dissensus, pour reprendre le terme de Jacques Rancière, en esquissant un monde sensible qui diffère du monde sensible existant. Grâce aux changements rapides et constants, la chorégraphie brouille les rapports 
et les situations des danseurs. Les règles de bienséance sont ainsi suspendues : «Tous ces petits prodiges s'opèrent si spontanément quand on aime la danse, qu'on n'a pas le temps de se raviser, et que la galerie n'a pas le temps de s'en apercevoir " (Sand, 1988, p. 221). En raison de ce relâchement de règles, la danse symbolise une situation où tout est possible. Un jour où Yseut parle aisément avec Pierre, ce dernier s'étonne de sa familiarité : "la voilà qui cause avec moi comme si nous étions à la contredanse » (p. 227). En réaménageant le temps, l'espace et les rapports humains, les danseurs s'émancipent de leurs situations tout en confondant le réel et l'irréel.

Pour conclure, Édouard et Le Compagnon du Tour de France portent une critique politique qui s'élabore dans les tentatives ratées de changements sociaux. Les moments dansants montrent pourtant la joie de nourrir et de poursuivre le rêve de renverser l'ordre social. Ces scènes de danse, ruptures radicales avec le temps du récit, accentuent le regret de ne pouvoir imaginer l'égalité que comme un rêve fugace. L'harmonie visible dans la communauté idéalisée créée par la danse est aussi éphémère que la danse elle-même. Même si cette société n'existe que dans des images passagères, ces brèves expériences d'une autre réalité influencent la façon dont les participants, les spectateurs et les lecteurs envisagent le présent ainsi que les avenirs possibles. La littérature et la danse utopiennes traitent de "visualiser des possibilités », non pas de les réaliser (Frye, 1967, p. 31).

\section{RÉFÉRENCES}

Baczko, B. (1989). Utopian Lights: The Evolution of the Idea of Social Progress (J. Greenberg, trad.). New York : Paragon House.

Cabanès, J.-L. (2009). La comédie du libéralisme dans Le Compagnon du Tour de France. Dans M. Watrelot et M. Hecquet (dir.), Le Compagnon du Tour de France de George Sand (p. 3140). Lille : Édition du Conseil Scientifique de l’Université Charles-de-Gaulle Lille 3.

Duras, C. (2007). Ourika. Édouard. Olivier ou le Secret. Paris : Gallimard.

Foucault, M. (1975). Pouvoir et corps. Quel corps ?, 2, 2-5.

Fourier, C. (1829). Le Nouveau Monde industriel et sociétaire, ou invention du procédé d'industrie attrayante et naturelle distribuée en séries passionnées. Paris : Bossange Père.

Frye, N. (1967). Varieties of Literary Utopias. Dans F. Manuel (dir.), Utopias and Utopian Thought (p. 25-49). Boston : Beacon Press.

Granger, S. (2019). Danser dans la France des Lumières. Rennes: Presses universitaires de Rennes. Guilcher, Y. (1998). La danse traditionnelle en France : d'une ancienne civilisation paysanne à un loisir revivaliste. Parthenay : FAMDT Éditions.

Giraudet, E. (1900). Traité de la danse (T. 2). Paris : s.n.

Marin, L. (1973). Utopiques : jeux d'espaces. Paris : Les Éditions de minuit.

Matinées sénonoises, ou proverbes françois. (1789). Paris : s.n.

More, T. (2003) Utopia (P. Turner, trad.). London : Penguin Books.

Nancy, J.-L. (2001). La Communauté affrontée. Paris : Galilée.

Ozouf, M. (1976). La Fête révolutionnaire. 1789-1799. Paris : Gallimard. 
Pailhès, G. (1910). La Duchesse de Duras et Chateaubriand d'après des documents inédits. Paris : Perrin.

Pauli, C. (1756). Elemens de la danse. Leipzig : Saalbach.

Rameau, P. (1748). Le Maître à danser. Paris : Rollin fils.

Rancière, J. (2011). Aisthesis : scènes du régime esthétique de l’art. Paris : Galilée.

Riot-Sarcey, M. (1998). Le réel de l'utopie. Essai sur le politique au XIXe siècle. Paris : Albin Michel.

Rousseau, J.-J. (1845). Julie, ou la nouvelle Héloïse. Paris : Charpentier.

Rousseau, J.-J. (1948). Les rêveries du promeneur solitaire. Genève : Librairie Droz.

Saint-Simon, L. (1830). Mémoires complets du duc de Saint-Simon sur le siècle de Louis XIV et la régence (T. 19). Paris : A. Sautelet.

Sand, G. (1988). Le Compagnon du Tour de France. Grenoble : Presses universitaires de Grenoble.

Schiller, F. (1971). Kallias oder über die Schönheit. Leipzig : Reclam.

Schor, N. (1993). George Sand and Idealism. New York : Columbia University Press.

Stendhal (1833). De L’Amour. Paris : Librairie universelle ancienne et moderne.

Sylvos, F. (2015). Utopie. Dans S. Bernard-Griffiths et P. Auraix-Jonchière (dir.), Dictionnaire de George Sand (p. 1216-1220). Paris : Honoré Champion.

Valéry, P. (2011). Philosophie de la danse. Dans C. Macel et E. Lavigne (dir.), Danser sa vie : écrits sur la danse (p. 83-94). Paris : Éditions du Centre Pompidou.

RÉSUMÉ : Durant le premier XIX ${ }^{\mathrm{e}}$ siècle, la contredanse, constituée de déplacements et d'interactions entre tous les danseurs n'importe leur place de départ, abolit les contraintes d'une société divisée en classes. Dans Édouard (1825), Claire de Duras compare le moment des contredanses, pendant un bal parisien de l'Ancien Régime, à une échappée vers l'Angleterre, où l'ascension sociale semble réalisable. De plus, la danse crée un espace où l'héroïne peut franchir les barrières entravant les membres de son sexe et empêchant le mariage par amour. George Sand, dans Le Compagnon du Tour de France (1840), contraste la possibilité de l'amour entre des personnages de classes différentes lors des contredanses avec l'impossibilité de ces unions dans la vie quotidienne. En établissant un non-lieu, les contredanses de ces romans produisent des moments éphémères où l'égalité et la liberté règnent, pourtant, hors de la danse, les hiérarchies sociales demeurent rigides.

Mots-clés : contredanses, utopie, George Sand, Claire de Duras, danses littéraires

\section{"It is as if we were in a contradance": choreographic utopias in novels by Claire de Duras and George Sand} ABSTRACT: During the first half of the 19th century, contradances, made up of displacements and interactions between all the dancers starting in different places, abolish the obligations imposed by a society divided into classes. In Édouard (1825), 
Claire de Duras compares the performance of contradances, during an ancien régime ball, to fleeing to England, where social ascension seems possible. George Sand, in Le Compagnon du Tour de France (1840), contrasts the possibility of romantic relationships across classes during contradances with the impossibility of these unions in everyday life. By establishing a non-place, these fictional dances create ephemeral moments during which equality and freedom reign; however, off the dance floor, social hierarchies remain rigid.

Keywords: contradances, utopia, George Sand, Claire de Duras, literary dances 